\title{
Chronic insomnia: cognitive behavioral and positive airway pressure therapies
}

Keywords: Chronic Insomnia, obstructive sleep apnea, upper airway resistant syndrome, sleep-disordered breathing
Volume 5 Issue 3 - 2018

\author{
Attapon Cheepsattayakorn, ${ }^{1,2}$ Ruangrong \\ Cheepsattayakorn ${ }^{3}$ \\ 'IOth Zonal Tuberculosis and Chest Disease Center,Thailand \\ ${ }^{2}$ Department of Disease Control, Ministry of Public Health, \\ Thailand \\ ${ }^{3}$ Department of Pathology, Chiang Mai University, Thailand
}

Correspondence: Attapon Cheepsattayakorn, I0th Zonal Tuberculosis and Chest Disease Center, I 43 Sridornchai Road Changklan Muang, Chiang Mai 50100, Thailand, Tel 66-53|40767/66-53-276364, Fax 66-53-|40773/66-53-273590, Email Attapon 1958@gmail.com, attaponche@yahoo.com

Received: June 09, 2018 | Published:June 14, 2018

\section{Editorial}

Chronic insomnia, a condition associated with increased mortality and healthcare cost is the most prevalent sleep disorder, globally. Widely extensive research has evaluated the etiology and economic impact, and evidence-based pharmacological and non-pharmacological therapies, but the optimal insomnia nosology has yet to be determined. Surprisingly and interestingly, a small corpus of recent research has studied on the associations between insomnia and obstructive sleep apnea (OSA) or upper airway resistant syndrome (UARS).Around one-third of community-dwelling older adults have clinical symptoms of insomnia and nearly one-third of them have undiagnosed sleepdisordered breathing (SDB). OSA, a common sleep breathing disorder affecting up to $17 \%$ of the middle-aged population and $2 \%$ to $7 \%$ of overall population, is characterized by periodic collapse of the upper airway during sleep. With a higher incidence of OSA with estimates of $14 \%$ to $55 \%$ in the Western world are associated with higher estimates of obesity. An OSA rate of $43 \%$ among older insomnia patients has been demonstrated. Increasing age further increases OSA prevalence. The prevalence of insomnia in OSA patients is estimated to be approximately $40 \%$ to $80 \%$. Rising obesity accompanying with increasingly ageing population further highlights the need for effective therapies of this sleep disorder. Several studies have revealed that $30 \%$ to $60 \%$ of OSA patients present with meaningfully clinical comorbid insomnia symptoms. A recent study among a large subjects of patients with chronic insomnia regularly using yet failing prescription or over-the-counter sleep aids, approximately $91 \%$ were objectively diagnosed with OSA or UARS. These study results are thought-provoking, a series of commentaries on causal associations between insomnia and SDB have been particularly provocative by raising questions about a potential bidirectional association between insomnia and SDB. The term "complex insomnia" has been proposed to categorized the comorbidity between chronic insomnia and OSA or UARS, after the unexpected finding of a $90 \%$ rate of objectively diagnosed sleep breathing disorders in a consecutive series of crime victims seeking therapy for insomnia and nightmares.
A wide body of research shows that cognitive behavioral therapy for insomnia (CBT-I) decreases sleep disturbance among older insomnia patients. Expansion of CBT-I training among mental health providers are underway. Several research studies revealed that CBT-I can be delivered over telephone and in condensed formats, for example: Brief Behavioral Treatment for Insomnia (BBT-I).The appropriate treatment sequence (concurrent versus sequential SDB treatment and CBT-I) for improving sleep disturbance among insomnia patients and comorbid SDB has only been investigated in a limited number of research studies, that revealed inconsistent results. Nasal cannula pressure transducer technology appears to yield the most accurate rates of complex insomnia. Unusual comorbidity between insomnia and SDB may require cognitive behavioral therapy for insomnia (CBT-I) or sedatives as well as positive airway pressure (PAP) therapy. Existence of insomnia has been demonstrated to negatively affect continuous positive airway pressure (CPAP) compliance due to possible pressure intolerance in some previous studies. Generally, conventional CPAP delivers higher pressure than necessary for much of the night as the needed CPAP pressure is selected based either on one night's titration (In a sleep laboratory) or during several nights at home and pressure requirements can vary considerably with sleep stage, sleeping posture, and environmental influences, such as sedative use and alcohol drinking. This problem can be addressed by Auto CPAP by continuously monitoring airflow changes and only increasing the pressure when the upper airway requires it. Generally, Auto $=$ CPAP delivers an overall lower mean treatment pressure than conventional CPAP. Nevertheless, there is limited evidence to indicate the improvement of CPAP compliance by using the AutoCPAP among unselected population, but the improvement might be different among OSA patients with insomnia. CPAP/AutoCPAP with SensAwake has been used in the general OSA population and has been demonstrated to provide the treatment efficacy at the lower overall pressure as CPAP/AutoCPAP without SensAwake. Nevertheless, there is no known published data on the use of SensAwake among the insomnia/ OSA population. Since 2000, at least nine studies revealed clearcut improvements of SDB after the use of PAP, nasal dilator strips, 
upper airway surgery, or bariatric surgery. One of these nine studies focused on PAP therapy sequentially applied after CBT-I. Another study with small sample of insomnia patients $(n=56)$ who previously failed and then ceased the use of standard forms of CPAP therapy were subsequently titrated with adaptive servo ventilation (ASV) after which $75 \%$ of them re-initiated PAP with this advanced technology. The majority of the 39 ASV users decreased in the insomnia severity index (ISI) were seen. (16.8 versus $9.7 ; p=0.001 ; d=1.40)$ after an average of 1.3 years of ASV use. The ubiquitous inability of patients with chronic insomnia disorder and comorbid OSA/UARS to adapt standard PAP devices, such as CPAP, CPAP with expiratory pressure relief (CFLEX), automatic CPAP (APAP), and bilevel PAP (BPAP) due to self-reported discomfort and intolerance triggered by the vaguely defined phenomenon of expiratory pressure intolerance (EPI) although among those using PAP devices with basic expiratory pressure relief(EPR) systems. These adverse effects were resolved by using ASV. Many patients with insomnia well adapted to autobilevel PAP (ABPAP) devices. Manually titrated, auto-adjusting algorithms embedded in these devices created a smoother airflow delivery system that in turn decreased insomnia patients' anxious responses to pressurized air. These results were consistent with previous research studies demonstrating the inadequacy of exclusive reliance on autotitrating algorithms in these devices.

In conclusion, these efforts that are providing will increase opportunities for patients to access CBT-I, that may in turn contribute to decreases in sleep disturbance among ageing adults.

\section{Acknowledgements}

None.

\section{Conflict of interest}

Author declares there is no conflict of interest in publishing the article. 